OPEN ACCESS

Edited by:

Linxin Li,

University of Oxford, United Kingdom

Reviewed by:

Tomi Petteri Laitinen,

Kuopio University Hospital, Finland

Marios K. Georgakis,

Hospital of the University of

Munich, Germany

${ }^{*}$ Correspondence:

Xianjia Ning

xjn0906@gmail.com

Qing Yang

yq1963884@sina.com

Jinghua Wang

jhw8799@yahoo.com

${ }^{\dagger}$ These authors have contributed equally to this work

Specialty section: This article was submitted to

Stroke,

a section of the journal

Frontiers in Neurology

Received: 13 November 2019 Accepted: 25 March 2020

Published: 24 April 2020

Citation:

Pan J, Liu J, Wang H, Li W, Du X, Lin Q, Zhang X, Qi D, Tu J, Ning $X$

Yang $Q$ and Wang J (2020)

Association of Carotid Atherosclerosis

With Lipid Components in

Asymptomatic Low-Income Chinese:

A Population-Based Cross-Sectional

Study. Front. Neurol. 11:276.

doi: 10.3389/fneur.2020.00276

\section{Association of Carotid Atherosclerosis With Lipid Components in Asymptomatic Low-Income Chinese: A Population-Based Cross-Sectional Study}

Jing Pan ${ }^{1 \dagger}$, Jie Liu ${ }^{1,2,3+}$, Hong Wang ${ }^{1,4 t}$, Weilan $\mathrm{Li}^{1}$, Xin Du ${ }^{5}$, Qiuxing Lin ${ }^{1,2,3}$, Xinxin Zhang ${ }^{6}$, Dongwang $Q i^{6}$, Jun $\mathrm{Tu}^{1,2,3}$, Xianjia Ning ${ }^{1,2,3 *}$, Qing Yang ${ }^{5 *}$ and Jinghua Wang ${ }^{1,2,3 *}$

${ }^{1}$ Department of Neurology, Tianjin Medical University General Hospital, Tianjin, China, ${ }^{2}$ Laboratory of Epidemiology, Tianjin Neurological Institute, Tianjin, China, ${ }^{3}$ Key Laboratory of Post-Neuroinjury Neuro-repair and Regeneration in Central Nervous System, Tianjin Neurological Institute, Tianjin, China, ${ }^{4}$ Tianjin Academy of Traditional Chinese Medicine Affiliated Hospital, Tianjin, China, ${ }^{5}$ Department of Cardiology, Tianjin Medical University General Hospital, Tianjin, China, ${ }^{6}$ Department of Endocrinology and Metabolism, Tianjin Medical University General Hospital, Tianjin, China

Intima-media thickness is a non-invasive arterial marker of early-stage atherosclerosis. Identifying carotid plaque is a superior surrogate endpoint for assessing atherosclerotic lesions. The aim of this study was to investigate the association of carotid intima-media thickness (CIMT) and carotid plaque with lipids among asymptomatic low-income rural residents in China. A total of 3,789 people aged $\geq 45$ years without a history of stroke or cardiovascular disease were recruited to this study. B-mode ultrasonography was performed to measure CIMT and identify carotid plaque for early identification of atherosclerosis. Multivariate analysis was used to assess the association of blood lipid levels with atherosclerosis. The mean CIMT across our cohort was $567 \mu \mathrm{m}$. A linear regression analysis showed that low-density lipoprotein cholesterol (LDL-C) and total cholesterol (TC) were risk factors for early-stage atherosclerosis; however, high-density lipoprotein cholesterol and triglycerides protected against early-stage atherosclerosis after adjusting for potential risk factors $(P<0.001)$. Carotid plaque risk increased by 24 and 62\% for each $1-\mathrm{mmol} / \mathrm{L}$ increase in TC and LDL-C $(P<0.001)$. These findings suggest that it is vital to manage and control the dyslipidemia standard levels in China, especially among rural residents, in order to reduce the burden of cardiovascular diseases.

Keywords: lipids, carotid intima-media thickness, carotid plaque, risk factors, ultrasonography, atherosclerosis

\section{INTRODUCTION}

Stroke was the leading cause of death and the third most common cause of disability in China between 1990 and $2017(1,2)$. The most common type of stroke is thrombotic stroke, and atherosclerosis is the most common cause (3). Thus, early detection and management of individuals at high risk is critical to prevent or delay otherwise inevitable end-events. 
Several studies have shown that intracranial or extracranial atherosclerosis is a valid predictor of the presence of a cerebrovascular disease (CVD) or stroke (4). The measurement of carotid intima media thickness (CIMT) using high-resolution ultrasound is useful for detecting early atherosclerotic changes $(5,6)$. Moreover, previous studies have demonstrated that increased CIMT was a marker for early atherosclerosis $(7,8)$ and that carotid plaque was a better parameter for assessing atherosclerotic lesions $(9,10)$. In addition, the hazard ratio of myocardial infarction or stroke was 1.09 for every 0.1$\mathrm{mm}$ increase in CIMT (11). Other studies have investigated the determinants of atherosclerosis, such as age, sex, current smoking, alcohol consumption, and education levels (12-15). The association of atherosclerosis with blood lipids has also been studied, but the results were controversial (16-18).

Considering the health consequences of atherosclerosis, further study of the effects of lipids on CIMT and carotid plaque is critical. However, to the best of our knowledge, few studies have examined this relationship, especially among low-income individuals with low educational levels who live in rural areas of China. Thus, we aimed to perform a population-based study to determine the association between blood lipids and different stages of atherosclerosis, including carotid plaque and CIMT, among a low-income population in rural China.

\section{METHODS}

\section{Participants and Study Design}

This was a cross-sectional, population-based survey conducted in the rural areas of Tianjin, China. The study was performed from April 2014 to January 2015 using participants from the Tianjin Brain Study, which has been described previously (19). In brief, the entire population comprised of 14,251 participants from 18 administrative villages in rural Tianjin, China. About $95 \%$ of the participants were low-income farmers, with a per capita disposable annual income of less than US\$1,600 in 2014 (20). All residents aged 45 years and older without CVDs were recruited to this study, while those with a history of CVD were excluded. Those with other vascular events were also excluded, including acute coronary events, any form of coronary artery disease, and peripheral artery disease. We determine CVD and other vascular events based on the patient's medical history and imaging data.

All investigations were approved by the ethics committee of Tianjin Medical University General Hospital. The study was carried out in accordance with the approved guidelines, and informed consent was obtained from all participants.

\section{Information Collection and Risk Factor Definitions}

All data in this study were obtained by trained epidemiological researchers through face-to-face interviews based on a prespecified questionnaire.

Demographic information, including name, sex, date of birth, and educational level, were retrieved from previous records. All participants were separated into four age groups: 45-54, 55-64, 65-74, and $\geq 75$ years. Educational level was classified into three groups according to the length of each individual's formal education: illiteracy (without education), 1-6 years, and $>6$ years.

Previous individual and family medical histories, which included hypertension, diabetes mellitus (DM), stroke, transient ischemic attack, and coronary heart disease, were obtained by patient self-reporting or review of previous records.

Lifestyle characteristics included cigarette smoking and alcohol consumption. Cigarette smoking was defined as smoking more than one cigarette per day for at least 1 year, and the participants were categorized as never smokers and smokers. Alcohol consumption was defined as drinking more than $500 \mathrm{~g}$ $\mathrm{f}$ alcohol per week for at least 1 year, and the participants were divided into a never alcohol consumption group and an alcohol consumption group.

\section{Physical Examination}

Blood pressure [including systolic blood pressure (SBP) and diastolic blood pressure (DBP)], height, weight, and circumference were measured at the local village clinic during the baseline survey. The levels of fasting blood glucose (FBG), total cholesterol (TC), triglycerides (TG), high-density lipoprotein cholesterol (HDL-C), and low-density lipoprotein cholesterol (LDL-C) in the serum were assessed at the Ji County People's Hospital. Carotid ultrasonography and 12-lead echocardiography were also conducted by a professional. Body mass index (BMI) was calculated as weight $(\mathrm{kg})$ divided by the square of height $\left(\mathrm{m}^{2}\right)$.

The sitting position BP was measured after abstaining from cigarettes, alcohol, tea, coffee, or physical activity for at least $30 \mathrm{~min}$. In an effort to minimize white coat hypertension, BP measurements were performed by the researchers in a quiet room, using the standard method described by the American Hypertension Association. Two readings were recorded and the average value was calculated.

\section{Ultrasonography Measurements}

One trained technician blinded to the participants' information performed the ultrasound examinations. The subjects were examined, while they were in a supine position, using B-mode ultrasonography (Terason 3000, Burlington, MA, USA) with a 5$12 \mathrm{MHz}$ linear array transducer. The bilateral extracranial carotid artery trees (including the common carotid artery, carotid sinus, and internal and external carotid arteries) were screened for plaque. Carotid plaque is defined as: (1) local CIMT exceeds $1.5 \mathrm{~mm}$, (2) localized CIMT bulge protrudes into the lumen for more than $0.5 \mathrm{~mm}$, and (3) local CIMT exceeds $50 \%$ in the peripheral medial lining; early-stage atherosclerosis was defined as CIMT $\geq 1 \mathrm{~mm}(21,22)$. CIMT at the near and far walls of the common carotid artery was measured on the left and the right, and three values were obtained: maximum CIMT, minimum CIMT, and average CIMT per lateral. The average CIMT was calculated according to the means for the sum of the CIMTs both on the left side and on the right side. The images were obtained and digitally stored according to a standard protocol (23). 


\section{Survey Procedure}

Local village physicians visited all eligible residents at their home according to a predefined protocol 1 day before the examination. We performed a physical examination (including blood pressure, weight, height, and circumference measurement, carotid ultrasonography, and 12-lead echocardiography) and collected blood samples at local village clinics between April and July in 2014. All blood samples were sent within $2 \mathrm{~h}$ of collection to the central laboratory at Tianjin Ji County People's Hospital for measurement of FBG, TC, TG, HDL-C, and LDL-C levels using standardized enzymic methods.

\section{Definition of Risk Factors}

Hypertension was defined as SBP $\geq 140 \mathrm{mmHg}, \mathrm{DBP} \geq 90 \mathrm{mmHg}$, or taking medication for hypertension. DM was defined as FBG concentration $\geq 7.0 \mathrm{mmol} / \mathrm{L}$ or taking medication for diabetes. Central obesity was defined as a waist circumference of $>102 \mathrm{~cm}$ in men and greater than $88 \mathrm{~cm}$ in women (24). Obesity was defined as a BMI $\geq 28.0 \mathrm{~kg} / \mathrm{m}^{2}$, and overweight was defined as a BMI of $24.0-27.9 \mathrm{~kg} / \mathrm{m}^{2}(25)$.

\section{Statistical Analyses}

Continuous variables, including age, TC, TG, HDL-C, and LDL$\mathrm{C}$, are presented as means and standard deviations; differences between groups are performed by Student's t-test. The categorical variables are presented as frequencies and 95\% confidence intervals (CIs); differences between groups are compared using chi-square tests. The risk factors for CIMT and CP were assessed individually for men and women using logistic regression analyses; the results of the univariate analysis were presented as unadjusted $\beta$ s (95\% CIs) or OR (95\% CIs). The association of lipids with CIMT and CP was performed by multivariate analysis; the results were presented as adjusted $\beta$ s (95\% CIs) or OR (95\% CIs) after adjustment for significant covariates in the univariate analysis. Of these covariates, education group, hypertension, DM, obesity, current smoking, and alcohol consumption were analyzed as categorical variables. Age, levels of FBP, TC, TG, HDL-C, and LDL-C were analyzed as continuous variables. $P<$ 0.05 was considered as statistically significant for dichotomous variables and categorical variables with three or four groups. SPSS for Windows (version 19.0; SPSS Inc., Chicago, IL, USA) was used for all analyses.

\section{RESULTS}

\section{Demographic Characteristics for Subjects}

A total of 4,012 participants were interviewed from among 5,380 residents aged 45 years and older during the study period, excluding 1,368 residents who were absent during the survey due to working outside $(n=931)$, separation of registered and actual residences $(n=349)$, and disability $(n=88)$. Finally, 3,789 individuals [1,560 men (41.2\%) and 2,229 women (58.8\%); mean age, 59.92 years] were enrolled in our study after excluding 223 residents with a previous history of CVD or stroke (Figure 1).

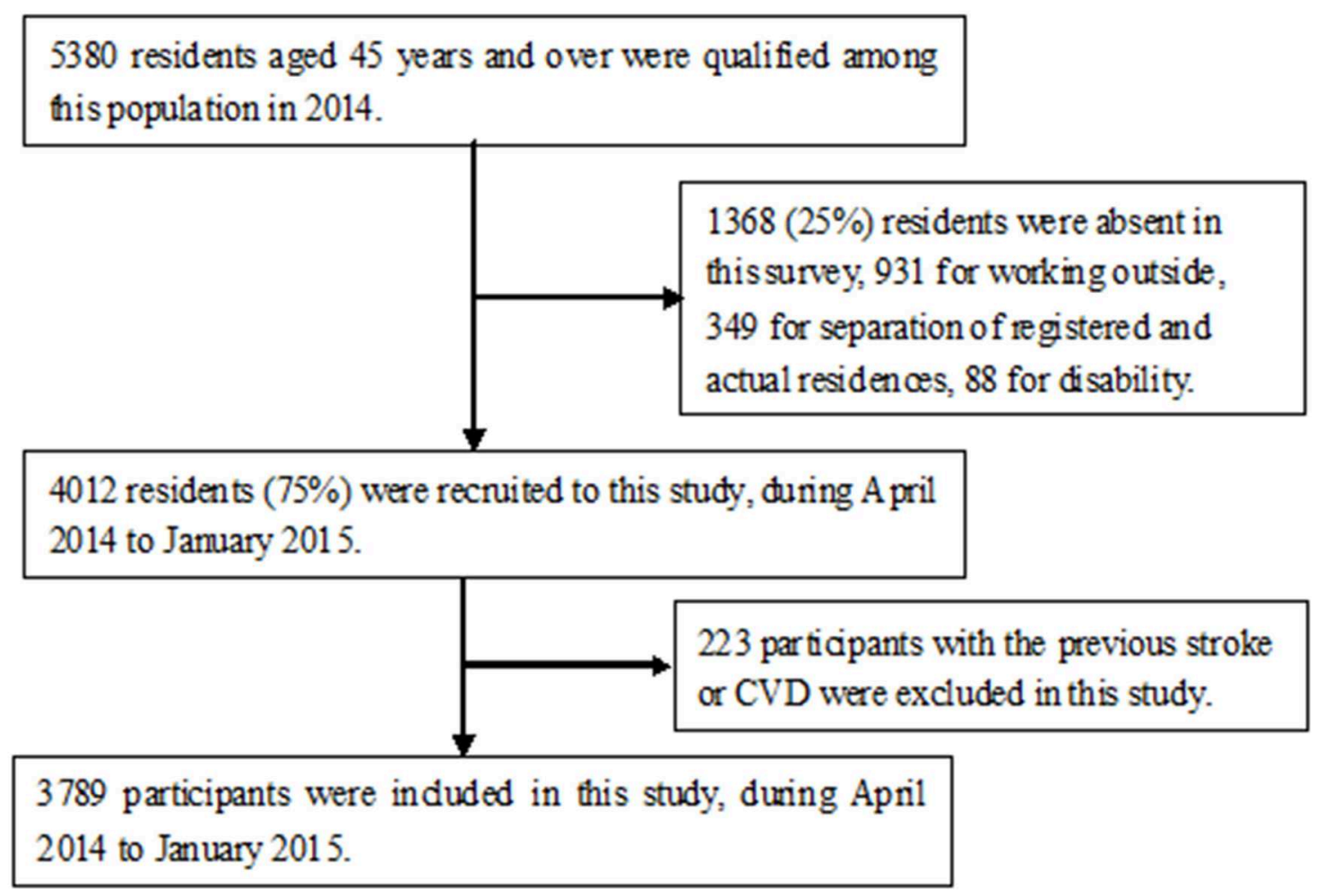

FIGURE 1 | Flow chart of participants. 
There were proportions of residents aged 45-54 years old among the absent group (57.2\%) than that in this study (32.6\%).

The education level of this population was very low; the average length of education was 5.48 years, and $17.4 \%$ of the participants had never received a formal education $(8.8 \%$ of men and $23.4 \%$ of women). The prevalence of hypertension, DM, central obesity, current smoking, and alcohol consumption was 20.4, 14.1, 35.8, 34.0, and 14.3\%, respectively. Moreover, the average SBP and DBP were high in this population, with mean values of 146.42 and $86.81 \mathrm{mmHg}$, respectively. The mean values of CIMT, TC, TG, HDL-C, and LDL-C were $567.13 \mu \mathrm{m}$, $4.87 \mathrm{mmol} / \mathrm{L}, 1.76 \mathrm{mmol} / \mathrm{L}, 1.46 \mathrm{mmol} / \mathrm{L}$, and $2.70 \mathrm{mmol} / \mathrm{L}$, respectively (Table $\mathbf{1}$ ).

\section{Association of Atherosclerosis With Conventional Risk Factors in the Univariate Analysis}

The mean CIMT and carotid plaque prevalence were higher in men than in women and increased with increasing age but decreased with advancing educational levels $(P<0.001)$. There was a significantly higher mean CIMT and carotid plaque prevalence in current smokers, those with hypertension and diabetes, than their opposite $(P<0.001)$. Alcohol consumers associated with mean CIMT and BMI associated with carotid plaque prevalence $(P<0.001$; Table 2$)$.

Moreover, CIMT increased with the increasing level of TC and LDL-C, while it decreased with the increasing level of TG and HDL-C. Moreover, the levels of TC and LDL-C were greater among the participants with carotid plaque than those without carotid plaque (Table 3).

\section{Association of Atherosclerosis With Lipids in the Multivariate Analysis}

Table 4 shows that the levels of TC, TG, HDL-C, and LDL-C associated independently with the mean CIMT after adjustment for other risk factors which are significant in the univariate analysis. The mean CIMT increased to $5.79 \mu \mathrm{m}$ for each 1$\mathrm{mmol} / \mathrm{L}$ increase of TC $(\beta, 5.79 ; 95 \% \mathrm{CI}, 3.04-8.55 ; P<0.001)$. Similarly, each $1-\mathrm{mmol} / \mathrm{L}$ increase of the LDL-C level resulted in a 5.07- $\mu \mathrm{m}$ increase of CIMT $(\beta, 5.07 ; 95 \% \mathrm{CI}, 2.93-7.21 ; P$ $<0.001)$. Oppositely, each 1-mmol/L increase of TG and HDLC levels resulted in 4.84- and 12.20- $\mu \mathrm{m}$ decrease of CIMT $(P<$ $0.001)$. Moreover, with each $1-\mathrm{mmol} / \mathrm{L}$ increase of TC and LDL$\mathrm{C}$, the risk of occurrence of carotid plaque increases by $24 \%$ (OR: 1.24, 95\% CI: $1.16-1.32 ; P<0.001$ ) and by $62 \%$ (OR: $1.62,95 \%$ CI: $1.51-1.73 ; P<0.001)$, respectively.

\section{DISCUSSION}

This is the first population-based study to explore the association between lipids and different stages of atherosclerosis among a Chinese rural population with a high incidence of stroke. An early manifestation of atherosclerosis was CIMT thickening, while plaque formation was a late sign of atherosclerosis. In this low-income population, the levels of TC, TG, HDL-C, and LDL-C associated significantly with mean CIMT. High levels
TABLE 1 | Characteristics of all participants in this study.

\begin{tabular}{|c|c|c|c|}
\hline Category & Total & Men & Women \\
\hline Total: & $3,789(100)$ & $1,560(41.2)$ & $2,229(58.8)$ \\
\hline Age, means (SD), years & $59.92(9.70)$ & $61.13(9.90)$ & $59.07(9.47)$ \\
\hline \multicolumn{4}{|l|}{ Age group, $n(\%)$} \\
\hline $45-54$ years & $1,236(32.6)$ & $430(27.6)$ & $806(36.2)$ \\
\hline $55-64$ years & $1,514(40.0)$ & $632(40.5)$ & $882(39.6)$ \\
\hline $65-74$ years & $724(19.1)$ & $338(21.7)$ & 386 (17.3) \\
\hline$\geq 75$ years & $315(8.3)$ & $160(10.3)$ & $155(7.0)$ \\
\hline Education, means (SD), years & $5.48(6.54)$ & $6.40(3.22)$ & $4.84(3.61)$ \\
\hline \multicolumn{4}{|l|}{ Education, $n(\%)$} \\
\hline 0 years & $659(17.4)$ & $137(8.8)$ & $522(23.4)$ \\
\hline $1-6$ years & $1,694(44.7)$ & $699(44.8)$ & 995 (44.6) \\
\hline$>6$ years & 1,436 (37.9) & $724(46.4)$ & $712(31.9)$ \\
\hline \multicolumn{4}{|l|}{ Smoking status, $n$ (\%) } \\
\hline Never smoking & $2,842(75.0)$ & $743(47.6)$ & 2099 (94.2) \\
\hline Ever smoking & $173(4.6)$ & $146(9.4)$ & $27(1.2)$ \\
\hline Current smoking & $774(20.4)$ & $671(43.0)$ & $103(4.6)$ \\
\hline \multicolumn{4}{|l|}{ Alcohol consumption, $n(\%)$} \\
\hline Never drinking & 3,198 (84.4) & $999(64.0)$ & 2199 (98.7) \\
\hline Ever drinking & $49(1.3)$ & $48(3.1)$ & $1(0.0)$ \\
\hline Current drinking & $542(14.3)$ & $513(32.9)$ & $29(1.3)$ \\
\hline Hypertension, n (\%) & $2,583(68.2)$ & $1,111(71.2)$ & $1,472(66.0)$ \\
\hline Diabetes, $n(\%)$ & $533(14.1)$ & $216(14.1)$ & $317(14.5)$ \\
\hline \multicolumn{4}{|l|}{ BMI, $n$ (\%) } \\
\hline Normal & $1,298(34.3)$ & $584(37.4)$ & $714(32.0)$ \\
\hline Overweight & $1,603(42.3)$ & 653 (41.9) & 950 (42.6) \\
\hline Obesity & $888(23.4)$ & $323(20.7)$ & $565(25.3)$ \\
\hline Central obesity, $n$ (\%) & $3,040(80.4)$ & $1,116(71.7)$ & $1,924(86.5)$ \\
\hline SBP, means (SD), mmHg & $146.42(22.17)$ & $147.76(21.41)$ & $145.49(22.64)$ \\
\hline DBP, means (SD), mmHg & $86.81(11.40)$ & 88.50 (11.22) & $85.62(11.39)$ \\
\hline BMI, means (SD), Kg/m² & $25.57(3.68)$ & $25.20(3.44)$ & $25.82(3.82)$ \\
\hline FBG, means (SD), mmol/L & $5.92(1.57)$ & $5.91(1.42)$ & $5.93(1.67)$ \\
\hline TC, means (SD), mmol/L & $4.87(1.09)$ & $4.62(1.00)$ & $5.04(1.11)$ \\
\hline TG, means (SD), mmol/L & $1.76(1.24)$ & $1.61(1.24)$ & $1.87(1.22)$ \\
\hline HDL-C, means (SD), mmol/L & $1.46(0.46)$ & $1.39(0.43)$ & $1.50(0.48)$ \\
\hline LDL-C, means (SD), mmol/L & $2.70(1.25)$ & $2.61(1.20)$ & $2.76(1.28)$ \\
\hline CIMT, means (SD), $\mu \mathrm{m}$ & 567 (87.92) & $583(92.64)$ & $556(82.59)$ \\
\hline
\end{tabular}

$S D$, standard deviation; SBP, systolic blood pressure; DBP, diastolic blood pressure; BMI, body mass index; FBG, fasting blood glucose; TC, total cholesterol; TG, triglycerides; $H D L-C$, high density lipoprotein cholesterol; $L D L-C$, low density lipoprotein cholesterol.

of TC and LDL-C and low levels of TG and HDL-C were the independent risk factors of early atherosclerosis. Nevertheless, an increase of the levels of TC and LDL-C associated with a higher risk of late-stage atherosclerosis.

There is overwhelming evidence that LDL-C is a causal contributor to the development of atherosclerosis (9). A previous study showed that LDL-C was a risk factor for the presence of any carotid plaque as well as for multiple plaques (16). Another study explored the correlation between blood lipid profile and atherosclerosis, finding that LDL-C was positively associated with the presence of carotid plaque (26). A large 
TABLE 2 | Relative factors of mean CIMT and carotid plaque prevalence in the univariate analysis.

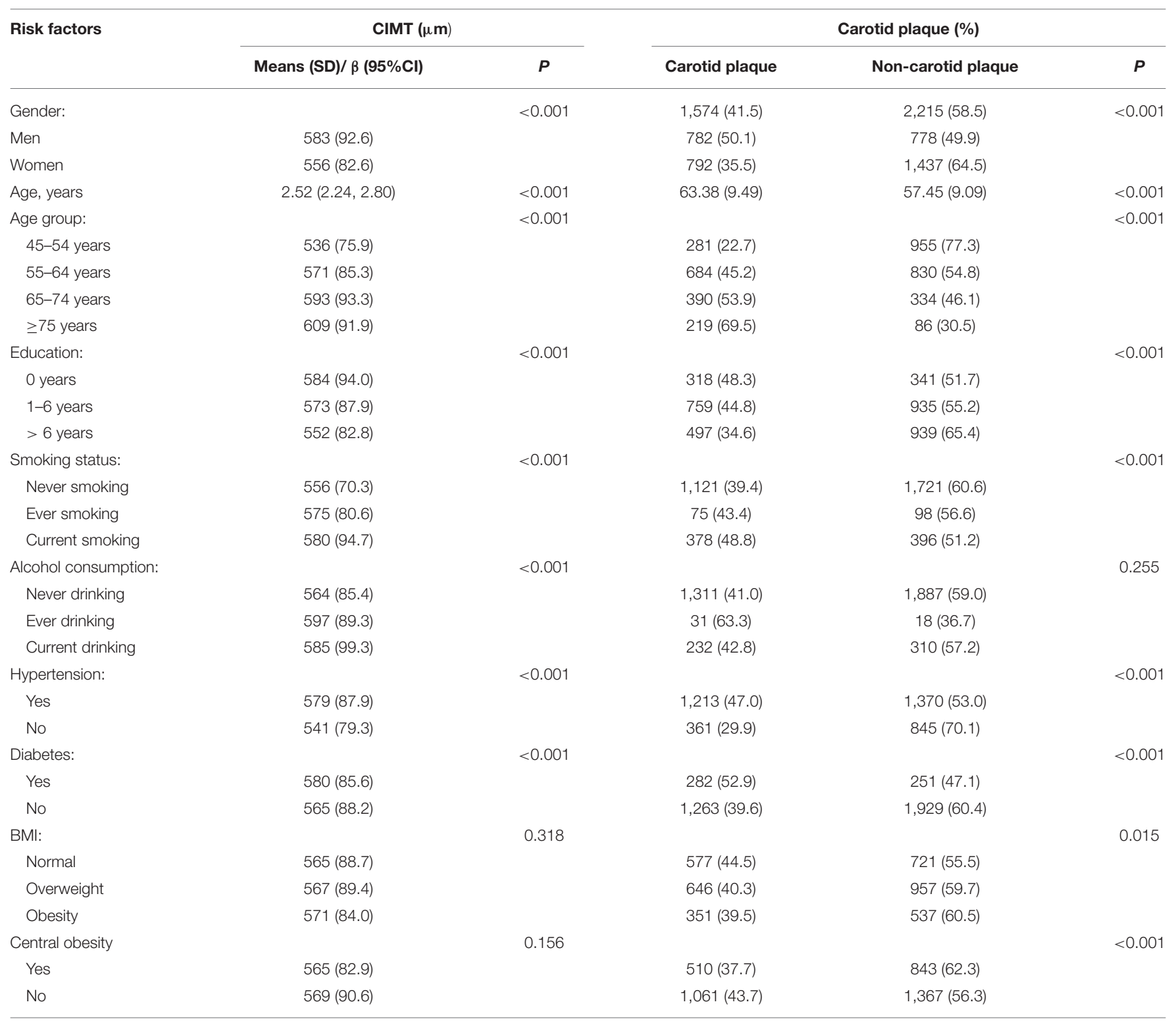

community-based cohort study suggested that elevated LDL-C was an independent predictor of carotid plaques among middleaged German women (27). Similar findings were observed in this study. The results of this present study suggested that the level of LDL-C was an independent risk factor for increasing CIMT, even after adjustment for other risk factors. In other words, LDL-C was determined as a risk factor not only to the formation but also to the development of atherosclerosis. We also identified elevated LDL-C as a major determinant of atherosclerosis progress, and the multivariate analysis confirmed the increase in carotid plaque risk with increasing LDL-C levels in participants who were 45 years and older. This study corroborated previous results that found serum LDL-C to be an important risk factor for atherosclerosis, particularly in middleaged individuals $(28,29)$. The underlying mechanism by which
LDL-C leads to arterial atherosclerosis is well established. Once the endothelial cell layer is damaged, LDL-C is deposited on the arterial wall, leading to the formation of fatty streaks and foam cells, a hallmark of atherosclerotic lesions $(30,31)$. This also explained the results that LDL affected the entire process of atherosclerosis.

A high level of plasma HDL-C has long been believed to be associated with a reduced number of cardiovascular events in the general population as well as in those at high risk for cardiovascular events $(32,33)$. One study from Italy demonstrated that the HDL-C levels were inversely associated with the development/presence of atherosclerosis in humans (34). In addition, two studies have shown a significant inverse relationship between HDL-C and CIMT $(35,36)$. However, other studies found no significant relationship between HDL-C 
TABLE 3 | Association of mean CIMT and carotid plaque with lipids in the univariate analysis.

\begin{tabular}{|c|c|c|c|c|c|}
\hline \multirow[t]{2}{*}{$\begin{array}{l}\text { Lipids } \\
\text { compon }\end{array}$} & \multicolumn{2}{|l|}{ CIMT $(\mu \mathrm{m})$} & \multicolumn{3}{|c|}{ Carotid plaque [means (SD)] } \\
\hline & $\beta(95 \% \mathrm{Cl})$ & $P$ & $\begin{array}{l}\text { Carotid } \\
\text { plaque }\end{array}$ & $\begin{array}{c}\text { Non-carotid } \\
\text { plaque }\end{array}$ & $\boldsymbol{P}$ \\
\hline TC & $3.40(0.80,5.99)$ & 0.010 & $4.99(1.15)$ & $4.78(1.04)$ & $<0.001$ \\
\hline $\mathrm{TG}$ & $-2.97(-5.25,-0.69)$ & 0.011 & $1.76(1.13)$ & $1.76(1.31)$ & 0.901 \\
\hline HDL-C & $-6.51(-12.65,-0.37)$ & 0.037 & $1.45(0.45)$ & $1.46(0.47)$ & 0.582 \\
\hline LDL-C & $6.57(4.32,8.82)$ & $<0.001$ & $3.07(1.44)$ & $2.43(1.02)$ & $<0.001$ \\
\hline
\end{tabular}

TABLE 4 | Association of mean CIMT and carotid plaque with lipids in the multivariate analysis.

\begin{tabular}{|c|c|c|c|c|c|}
\hline \multirow[t]{2}{*}{$\begin{array}{l}\text { Lipids } \\
\text { compone }\end{array}$} & \multicolumn{2}{|l|}{ CIMT $(\mu \mathrm{m})$} & \multicolumn{3}{|c|}{ Carotid plaque } \\
\hline & $\beta(95 \% \mathrm{Cl})$ & $P$ & References & $\begin{array}{c}\text { OR } \\
(95 \% \mathrm{Cl})\end{array}$ & $P$ \\
\hline TC & $5.79(3.04,8.55)$ & $<0.001$ & - & $\begin{array}{c}1.24(1.15, \\
1.36)\end{array}$ & $<0.001$ \\
\hline TG & $-4.84(-7.26,-2.42)$ & $<0.001$ & - & - & - \\
\hline HDL-C & $-12.20(-18.61,-5.79)$ & $<0.001$ & - & - & - \\
\hline LDL-C & $5.07(2.93,7.21)$ & $<0.001$ & - & $\begin{array}{c}1.62(1.51, \\
1.73)\end{array}$ & $<0.001$ \\
\hline
\end{tabular}

Model CIMT: including TC, TG, HDL-C, and LDL-C adjusted by statistical significance variates in the univariate analysis. Model carotid plaque: including TC and LDL-C adjusted by significant variates in the univariate analysis.

and $\operatorname{CIMT}(6,37,38)$, including one restricted to diabetic patients (6). In contrast to the aforementioned reports, our results suggested that high $\mathrm{HDL}-\mathrm{C}$ was an independent protective factor against increased CIMT, but no significant association between the presence of carotid plaque and HDL-C was observed. Thus, HDL can delay the formation of atherosclerosis, but there was no effect to progress in atherosclerosis. Several possible mechanisms may explain this association between HDL-C and early-stage atherosclerosis. HDL-C is believed to inhibit the formation of atherosclerosis lesions in the arterial wall by removing excess cholesterol from cells and preventing cell adhesion and transmigration (39). These mechanisms may suggest that there is increased thickening of the arterial wall in individuals with low plasma HDL-C levels (40). However, the mechanisms underlying HDL-C's effect on the carotid plaque remain unclear.

While numerous previous studies have focused on CIMT, few have reported the effects of TC and TG on CIMT. Furthermore, the relationship between TG and CIMT remains controversial. The main finding of a study from Germany was that fasting TG was independently and positively associated with mean CIMT in acute ischemic stroke patients (41). Others have found no association between CIMT and fasting (42-44) or post-challenge TG (45). The present study demonstrated that CIMT increased with TC increase and TG decrease, while the risk of the carotid plaque increased with TC increase, but there were no significant differences between the carotid plaque and the LDL-C levels. In this study, although CIMT decreased with TG increase, there were no significant differences between TG and carotid plaque. Moreover, there was a thin CIMT in this low-income population, with a mean value of $567 \mu \mathrm{m}$. The precise relationship between CIMT and TG level needs to be explored by a long-term follow-up.

There are several limitations to this study. First, considering our study design, the inherent limitation of a cross-sectional study is that it cannot validate causal links between significant variables and CIMT; thus, further longitudinal studies are needed in order to determine causality. Second, the reliance on Chinese rural residents with low income and education levels may limit the generalizability of the study's findings. However, this rural population accounts for more than $50 \%$ of the total population of China; therefore, our results represent the findings in a significant population. In addition, we did not collect all information on medicine in all participants; however, due to their disadvantaged socioeconomic status, the frequency of medicine use was low and would not impact the validity of the results.

\section{CONCLUSIONS}

In conclusion, the levels of TC, TG, HDL-C, and LDL-C associated significantly with the mean CIMT. High levels of TC and LDL-C and low levels of TG and HDL-C were the independent risk factors of early atherosclerosis. Nevertheless, increase in the levels of TC and LDL-C associated with a higher risk of late-stage atherosclerosis. These findings suggest that it is vital to manage and control the dyslipidemia standard levels in China, especially among rural residents, in order to reduce the burden of CVD.

\section{DATA AVAILABILITY STATEMENT}

The datasets analyzed in this article are not publicly available. Requests to access the datasets should be directed to Jinghua Wang,jhw8799@yahoo.com.

\section{ETHICS STATEMENT}

The studies involving human participants were reviewed and approved by the ethics committee of Tianjin Medical University General Hospital; the methods were carried out in accordance with the approved guidelines, and informed consent was obtained from all participants. The patients/participants provided their written informed consent to participate in this study.

\section{AUTHOR CONTRIBUTIONS}

JW, QY, and XN contributed to the study design, performed the data interpretation, and critical review. JW performed the data analysis. JP, JL, and HW contributed to the drafting of the article. 
JP, JL, HW, WL, XD, QL, XZ, DQ, JT, XN, and JW performed the data collection, case diagnoses, and confirmation of case diagnoses. All the authors read, revised, and approved the final version of the paper.

\section{ACKNOWLEDGMENTS}

We thank all the participants of the Tianjin Brain Study and the local medical care professionals for their valuable contributions.

\section{REFERENCES}

1. Zhou M, Wang H, Zeng X, Yin P, Zhu J, Chen W, et al. Mortality, morbidity, and risk factors in China and its provinces, 1990-2017: a systematic analysis for the Global Burden of Disease Study 2017. Lancet. (2019) 394:114558. doi: 10.1016/S0140-6736(19)30427-1

2. Wang J, Bai L, Shi M, Yang L, An Z, Li B, et al. Trends in age of first-ever stroke following increased incidence and life expectancy in a low-income chinese population. Stroke. (2016) 47:929-35. doi: 10.1161/STROKEAHA.115.012466

3. Yueniwati Y, Darmiastini NK, Arisetijono E. Thicker carotid intimamedia thickness and increased plasma VEGF levels suffered by post-acute thrombotic stroke patients. Int J Gen Med. (2016) 9:447-52. doi: 10.2147/IJGM.S114577

4. Oh PC, Han SH, Kim JS, Park JB, Koh KK. Carotid plaque in absence of traditional and non-traditional cardiovascular risk factors. Int J Cardiol. (2010) 143:e57-9. doi: 10.1016/j.ijcard.2008.12.049

5. Iglesias del Sol A, Bots ML, Grobbee DE, Hofman A, Witteman JC. Carotid intima-media thickness at different sites: relation to incident myocardial infarction; The Rotterdam Study. Eur Heart J. (2002) 23:93440. doi: 10.1053/euhj.2001.2965

6. Yamasaki Y, Kodama M, Nishizawa H, Sakamoto K, Matsuhisa M, Kajimoto Y, et al. Carotid intima-media thickness in Japanese type 2 diabetic subjects: predictors of progression and relationship with incident coronary heart disease. Diabetes Care. (2000) 23:1310-5. doi: 10.2337/diacare.23.9.1310

7. Lorenz MW, Markus HS, Bots ML, Rosvall M, Sitzer M. Prediction of clinical cardiovascular events with carotid intima-media thickness: a systematic review and meta-analysis. Circulation. (2007) 115:45967. doi: 10.1161/CIRCULATIONAHA.106.628875

8. Kablak-Ziembicka A, Tracz W, Przewlocki T, Pieniazek P, Sokolowski A, Konieczynska M. Association of increased carotid intima-media thickness with the extent of coronary artery disease. Heart. (2004) 90:128690. doi: 10.1136/hrt.2003.025080

9. Kuo F, Gardener H, Dong C, Cabral D, Della-Morte D, Blanton $\mathrm{SH}$, et al. Traditional cardiovascular risk factors explain the minority of the variability in carotid plaque. Stroke. (2012) 43:1755-60. doi: 10.1161/STROKEAHA.112.651059

10. Naqvi TZ, Lee MS. Carotid intima-media thickness and plaque in cardiovascular risk assessment. JACC Cardiovasc Imaging. (2014) 7:102538. doi: 10.1016/j.jcmg.2013.11.014

11. Den Ruijter HM, Peters SA, Anderson TJ, Britton AR, Dekker JM, Eijkemans MJ, et al. Common carotid intima-media thickness measurements in cardiovascular risk prediction: a meta-analysis. JAMA. (2012) 308:796803. doi: 10.1001/jama.2012.9630

12. Su TC, Chien KL, Jeng JS, Chen MF, Hsu HC, Torng PL, et al. Age- and gender-associated determinants of carotid intima-media thickness: a community-based study. J Atheroscler Thromb. (2012) 19:872-80. doi: 10.5551/jat.10728

13. Tattersall MC, Gassett A, Korcarz CE, Gepner AD, Kaufman JD, Liu KJ, et al. Predictors of carotid thickness and plaque progression during a decade: the Multi-Ethnic Study of Atherosclerosis. Stroke. (2014) 45:325762. doi: 10.1161/STROKEAHA.114.005669

14. Debus ES. Commentary on 'carotid artery atherosclerosis among 65-year-old Swedish men: a population based screening study'. Eur J Vasc Endovasc Surg. (2014) 48:11-2. doi: 10.1016/j.ejvs.2014.04.013

15. Yang D, Iyer S, Gardener H, Della-Morte D, Crisby M, Dong C, et al. Cigarette smoking and carotid plaque echodensity in the Northern Manhattan Study. Cerebrovasc Dis. (2015) 40:136-43. doi: 10.1159/000434761

16. Fan AZ, Dwyer JH. Sex differences in the relation of HDL cholesterol to progression of carotid intima-media thickness: the Los Angeles Atherosclerosis Study. Atherosclerosis. 195:e191-6. doi: 10.1016/j.atherosclerosis.2007.03.045

17. Taniwaki H, Kawagishi T, Emoto M, Shoji T, Kanda H, Maekawa K, et al. Correlation between the intima-media thickness of the carotid artery and aortic pulse-wave velocity in patients with type 2 diabetes. Vessel wall properties in type 2 diabetes. Diabetes Care. (1999) 22:18517. doi: $10.2337 /$ diacare.22.11.1851

18. Katakami N, Kaneto H, Osonoi T, Saitou M, Takahara M, Sakamoto F, et al. Usefulness of lipoprotein ratios in assessing carotid atherosclerosis in Japanese type 2 diabetic patients. Atherosclerosis. (2011) 214:4427. doi: 10.1016/j.atherosclerosis.2010.10.035

19. Zhan C, Shi M, Yang Y, Pang H, Fei S, Bai L, et al. Prevalence and risk factors of carotid plaque among middle-aged and elderly adults in rural Tianjin, China. Sci Rep. (2016) 6:23870. doi: 10.1038/srep23870

20. Wang J, Ning X, Yang L, Tu J, Gu H, Zhan C, et al. Sex differences in trends of incidence and mortality of first-ever stroke in rural Tianjin, China, from 1992 to 2012. Stroke. (2014) 45:1626-31. doi: 10.1161/STROKEAHA.113.003899

21. Lutsey PL, Diez Roux AV, Jacobs DR Jr, Burke GL, Harman J, Shea S, et al. Associations of acculturation and socioeconomic status with subclinical cardiovascular disease in the multi-ethnic study of atherosclerosis. Am J Public Health. (2008) 98:1963-70. doi: 10.2105/AJPH.2007.123844

22. Zhang Y, Bai L, Shi M, Lu H, Wu Y, Tu J, et al. Features and risk factors of carotid atherosclerosis in a population with high stroke incidence in China. Oncotarget. (2017) 8:57477-88. doi: 10.18632/oncotarget.15415

23. Touboul PJ, Hennerici MG, Meairs S, Adams H, Amarenco P, Bornstein $\mathrm{N}$, et al. Mannheim carotid intima-media thickness and plaque consensus (2004-2006-2011). An update on behalf of the advisory board of the 3rd, 4 th and 5th watching the risk symposia, at the 13th, 15th and 20th European Stroke Conferences, Mannheim, Germany, 2004, Brussels, Belgium, 2006, and Hamburg, Germany, 2011. Cerebrovasc Dis. (2012) 34:2906. doi: 10.1159/000343145

24. Parikh RM, Joshi SR, Pandia K. Index of central obesity is better than waist circumference in defining metabolic syndrome. Metab Syndr Relat Disord. (2009) 7:525-7. doi: 10.1089/met.2008.0102

25. Zhou BF. Effect of body mass index on all-cause mortality and incidence of cardiovascular diseases-report for meta-analysis of prospective studies open optimal cut-off points of body mass index in Chinese adults. Biomed Environ Sci. (2002) 15:245-52. doi: 10.1016/S0006-3207(02)00045-9

26. Yang C, Sun Z, Li Y, Ai J, Sun Q, Tian Y. The correlation between serum lipid profile with carotid intima-media thickness and plaque. BMC Cardiovasc Disord. (2014) 14:181. doi: 10.1186/1471-2261-14-181

27. Sinning C, Wild PS, Echevarria FM, Wilde S, Schnabel R, Lubos $\mathrm{E}$, et al. Sex differences in early carotid atherosclerosis (from the community-based Gutenberg-Heart Study). Am J Cardiol. (2011) 107:18417. doi: 10.1016/j.amjcard.2011.02.318

28. Su TC, Lee YT, Chou S, Hwang WT, Chen CF, Wang JD. Twenty-four-hour ambulatory blood pressure and duration of hypertension as major determinants for intima-media thickness and atherosclerosis of carotid arteries. Atherosclerosis. (2006) 184:151-6. doi: 10.1016/j.atherosclerosis.2005.03.041

29. Yang KC, Chen MF, Su TC, Jeng JS, Hwang BS, Lin LY, et al. Hepatitis B virus seropositivity is not associated with increased risk of carotid atherosclerosis in Taiwanese. Atherosclerosis. (2007) 195:3927. doi: 10.1016/j.atherosclerosis.2006.10.018

30. Conti P, Shaik-Dasthagirisaeb Y. Atherosclerosis: a chronic inflammatory disease mediated by mast cells. Cent Eur J Immunol. (2015) 40:3806. doi: 10.5114/ceji.2015.54603

31. Glass CK, Witztum JL. Atherosclerosis. the road ahead. Cell. (2001) 104:50316. doi: 10.1016/S0092-8674(01)00238-0 
32. Emerging Risk Factors Collaboration, Di Angelantonio E, Sarwar N, Perry P, Kaptoge S, Ray KK, et al. Major lipids, apolipoproteins, and risk of vascular disease. JAMA. (2009) 302:1993-2000. doi: 10.1001/jama.2009.1619

33. Zhang L, Qiao Q, Tuomilehto J, Hammar N, Ruotolo G, Stehouwer CD, et al. The impact of dyslipidaemia on cardiovascular mortality in individuals without a prior history of diabetes in the DECODE Study. Atherosclerosis. (2009) 206:298-302. doi: 10.1016/j.atherosclerosis.2008.12.043

34. Baldassarre D, Amato M, Pustina L, Tremoli E, Sirtori CR, Calabresi L, et al. Increased carotid artery intima-media thickness in subjects with primary hypoalphalipoproteinemia. Arterioscler Thromb Vasc Biol. (2002) 22:31722. doi: $10.1161 /$ hq0202.104848

35. Wallenfeldt K, Bokemark L, Wikstrand J, Hulthe J, Fagerberg B. Apolipoprotein B/apolipoprotein A-I in relation to the metabolic syndrome and change in carotid artery intima-media thickness during 3 years in middle-aged men. Stroke. (2004) 35:2248-52. doi: 10.1161/01.STR.0000140629.65145.3c

36. Stensland-Bugge E, Bønaa KH, Joakimsen O, Njølstad I. Sex differences in the relationship of risk factors to subclinical carotid atherosclerosis measured 15 years later : the Tromsø study. Stroke. (2000) 31:57481. doi: 10.1161/01.STR.31.3.574

37. Mackinnon AD, Jerrard-Dunne P, Sitzer M, Buehler A, von Kegler S, Markus HS. Rates and determinants of site-specific progression of carotid artery intima-media thickness: the carotid atherosclerosis progression study. Stroke. (2004) 35:2150-4. doi: 10.1161/01.STR.0000136720.21095.f3

38. Fujii K, Abe I, Ohya Y, Ohta Y, Arima H, Akasaki T, et al. Risk factors for the progression of early carotid atherosclerosis in a male working population. Hypertens Res. (2003) 26:465-71. doi: 10.1291/hypres.26.465

39. Cockerill GW, Rye KA, Gamble JR, Vadas MA, Barter PJ. Highdensity lipoproteins inhibit cytokine-induced expression of endothelial cell adhesion molecules. Arterioscler Thromb Vasc Biol. (1995) 15:198794. doi: 10.1161/01.ATV.15.11.1987

40. Wilt TJ, Rubins HB, Robins SJ, Riley WA, Collins D, Elam M, et al. Carotid atherosclerosis in men with low levels of HDL cholesterol. Stroke. (1997) 28:1919-25. doi: 10.1161/01.STR.28.10.1919
41. Batluk J, Leonards CO, Grittner U, Lange KS, Schreiber SJ, Endres M, et al. Triglycerides and carotid intima-media thickness in ischemic stroke patients. Atherosclerosis. (2015) 243:186-91. doi: 10.1016/j.atherosclerosis.2015. 09.003

42. Whincup PH, Nightingale CM, Owen CG, Rapala A, Bhowruth DJ, Prescott $\mathrm{MH}$, et al. Ethnic differences in carotid intima-media thickness between UK children of black African-Caribbean and white European origin. Stroke. (2012) 43:1747-54. doi: 10.1161/STROKEAHA.111.644955

43. Polak JF, Person SD, Wei GS, Godreau A, Jacobs DR Jr, Harrington A, et al. Segment-specific associations of carotid intima-media thickness with cardiovascular risk factors: the Coronary Artery Risk Development in Young Adults (CARDIA) study. Stroke. (2010) 41:9-15. doi: 10.1161/STROKEAHA.109.566596

44. Lind L, Wohlin M, Andren B, Sundström J. The echogenicity of the intimamedia complex in the common carotid artery is related to insulin resistance measured by the hyperinsulinemic clamp in elderly men. Clin Physiol Funct Imaging. (2013) 33:137-42. doi: 10.1111/cpf.12006

45. Alssema M, Schindhelm RK, Dekker JM, Diamant M, Kostense PJ, Teerlink $\mathrm{T}$, et al. Postprandial glucose and not triglyceride concentrations are associated with carotid intima media thickness in women with normal glucose metabolism: the Hoorn prandial study. Atherosclerosis. (2008) 196:7129. doi: 10.1016/j.atherosclerosis.2006.12.021

Conflict of Interest: The authors declare that the research was conducted in the absence of any commercial or financial relationships that could be construed as a potential conflict of interest.

Copyright (C) 2020 Pan, Liu, Wang, Li, Du, Lin, Zhang, Qi, Tu, Ning, Yang and Wang. This is an open-access article distributed under the terms of the Creative Commons Attribution License (CC BY). The use, distribution or reproduction in other forums is permitted, provided the original author(s) and the copyright owner(s) are credited and that the original publication in this journal is cited, in accordance with accepted academic practice. No use, distribution or reproduction is permitted which does not comply with these terms. 